\title{
CSF Removal in Infantile Posthemorrhagic Hydrocephalus Results in Significant Improvement in Cerebral Hemodynamics
}

\author{
JANET S. SOUL, ERIC EICHENWALD, GENE WALTER, JOSEPH J. VOLPE, AND \\ ADRÉ J. DU PLESSIS \\ Department of Neurology, Children's Hospital [J.S.S., G.W., J.J.V., A.J.D.], and Department of Newborn \\ Medicine, Brigham and Women's Hospital and Harvard Medical School [E.E.], Boston, Massachusetts
}

02115 , U.S.A.

\section{ABSTRACT}

Rational intervention in infants with posthemorrhagic hydrocephalus $(\mathrm{PHH})$ would be facilitated greatly by bedside measure of impaired cerebral perfusion, as there is substantial evidence that impaired perfusion and oxidative metabolism contribute to irreversible brain injury in hydrocephalus. Near-infrared spectroscopy (NIRS) measures changes in the cerebral concentration of oxygenated and deoxygenated hemoglobin and oxidized cytochrome oxidase at the bedside of infants continuously and noninvasively. The total hemoglobin and the hemoglobin difference signal are derived from the sum and difference, respectively, of oxygenated and deoxygenated hemoglobin. Changes in total hemoglobin reflect changes in cerebral blood volume; our previous work has shown that changes in hemoglobin difference signal reflect changes in cerebral blood flow. We hypothesized that cerebrospinal fluid (CSF) removal in infants with $\mathrm{PHH}$ would result in significant increases in cerebral perfusion, cerebral blood volume, and oxidative metabolism, as measured by NIRS. Continuous NIRS recordings were performed during CSF removal on 16 infants with $\mathrm{PHH}$. There was a statistically significant increase in oxygenated hemoglobin $(p<0.001)$, total hemoglobin $(p=0.001)$, and hemoglobin difference signal ( $p=$ 0.006 ), but not oxidized cytochrome oxidase, accompanying
$\mathrm{CSF}$ removal. There was no significant correlation between either the volume of CSF removed (in milliliters per kilogram body weight) or the opening pressure and the change in any of the measured or calculated NIRS signals. These findings demonstrate the pronounced effect of CSF removal on cerebral perfusion in infants with PHH. NIRS may be a useful technique to detect impending cerebral ischemia in such infants and thereby provide a means to guide the rational management of $\mathrm{PHH}$. (Pediatr Res 55: 872-876, 2004)

Abbreviations
IVH, intraventricular hemorrhage
PHH, posthemorrhagic hydrocephalus
NIRS, near-infrared spectroscopy
HbO, oxygenated hemoglobin
Hb, deoxygenated hemoglobin
HbD, hemoglobin difference signal
HbT, total hemoglobin
CytOx, oxidized cytochrome aa ${ }_{3}$
CBF, cerebral blood flow
CBV, cerebral blood volume
CSF, cerebrospinal fluid

Very low birth weight infants are at high risk of developing IVH (1). PHH is a major complication of IVH and contributes importantly to subsequent long-term cognitive and motor disability (2-5). The management of infants with PHH is controversial because the benefits of any specific treatment regimen have not been established $(2,5-8)$. However, substantial data from experimental animal studies of hydrocephalus suggest

Received August 14, 2003; accepted September 18, 2003

Correspondence: Adré J. du Plessis, M.D., Children's Hospital, Department of Neurology, Fegan 11, 300 Longwood Avenue, Boston, MA 02115, U.S.A.; e-mail: adre.duplessis@childrens.harvard.edu

Supported by the Charles H. Hood Foundation (Boston, MA, U.S.A.), the United Cerebral Palsy Foundation, the William Randolph Hearst Foundation, the Charles A Dana Foundation, and the Lifebridge Fund.

DOI: 10.1203/01.PDR.0000119370.21770.AC that impairments in cerebral hemodynamics and oxidative metabolism play a significant role in the pathophysiology of brain injury associated with this disorder (1). Animal studies have clearly shown that early (but not late) treatment of hydrocephalus results in a reversal of impaired cerebral perfusion and oxidative metabolism and the prevention of brain injury and cognitive impairment (9-13). Consistent with the animal studies, limited studies of human infants suggest decreased cerebral perfusion and increased cerebrovascular resistance with $\mathrm{PHH}(1,14,15)$. However, the previous investigations have used methods that are not applicable serially and at the bedside (e.g. positron emission tomography) or that do not measure cerebral perfusion (e.g. Doppler ultrasound). Thus, there is a need for a technique that can determine whether an 
infant with PHH has impaired cerebral perfusion. Such a method could facilitate rational decision making in the treatment of PHH and thereby prevention of secondary brain injury.

NIRS is a portable noninvasive technique that can be used to measure changes in cerebral hemodynamics at the bedside of infants. Two experimental studies have shown that changes in CBF correlate with changes in the HbD (see "Methods") measured by NIRS $(16,17)$. Thus, we aimed to use this technique to determine whether treatment of PHH by CSF removal resulted in changes in cerebral hemodynamics and oxidative metabolism. We hypothesized that CSF removal in infants with $\mathrm{PHH}$ would result in significant increases in cerebral perfusion, $\mathrm{CBV}$, and oxidative metabolism.

\section{METHODS}

Subjects. Newborn infants with IVH who exhibited progressive enlargement of the lateral ventricles (identified by serial cranial ultrasound scans) were eligible for this study. Informed consent was obtained from the parent(s) for all subjects. The study was approved by the institutional review boards at the two participating institutions (Children's Hospital and Brigham and Women's Hospital, Boston, MA, U.S.A.).

NIRS measurements. NIRS was used to detect changes in cerebral hemodynamics and oxygenation continuously and noninvasively at the bedside of the infant (18). NIRS is based on the principle that hemoglobin and CytOx (the terminal enzyme in the mitochondrial respiratory chain) are naturally occurring chromophores that absorb near-infrared light as a function of their oxygenation state. NIRS measures quantitative changes in $\mathrm{HbO}$ and $\mathrm{Hb}$ and thereby provides information about intravascular oxygenation. Changes in the redox state of CytOx reflect alterations in intracellular oxygenation.

NIRS measurements were collected using a four-wavelength $(775,824,843$, and $909 \mathrm{~nm})$ spectrophotometer (NIRO500, Hamamatsu Photonics, Hamamtsu City, Japan). The instrument transmits light generated by four laser diodes via a fiberoptic bundle to an optode placed on the infant's head. An identical fiberoptic bundle transmits the light received by a second optode to the photomultiplier tube where the photons are counted. NIRS optodes were placed on the skin overlying the frontoparietal cerebrum with an interoptode spacing of 3.5 $\mathrm{cm}$. The optodes were covered with opaque material and secured to the head with an elastic nonadhesive bandage.

The changes in near-infrared light absorption were used to calculate the changes in the relative concentration in micromoles per liter of each chromophore ( $\mathrm{Hb}, \mathrm{HbO}$, and CytOx) from an arbitrary baseline value, according to the BeerLambert law $(19,20)$. The calculations were corrected for the interoptode distance of $3.5 \mathrm{~cm}$. The differential pathlength factor corrects the total pathlength of the light for the degree of scattering within the tissue sample. For this study, the differential pathlength factor was assumed to be 4.39 as previously determined in human infants (21). Thus, changes in the concentration of NIRS signals were expressed in units of micromoles per liter. The NIRS instrument simultaneously recorded the peripheral oxygen saturation with the NIRS signals noted above. The $\mathrm{HbT}$ and the $\mathrm{HbD}$ were calculated from the sum
$(\mathrm{HbO}+\mathrm{Hb})$ and difference $(\mathrm{HbO}-\mathrm{Hb})$, respectively, of $\mathrm{HbO}$ and $\mathrm{Hb}$.

Study protocol. NIRS optodes were placed on the frontoparietal regions of the infants' heads before CSF removal, as noted above. The NIRS signals ( $\mathrm{Hb}, \mathrm{HbO}, \mathrm{CytOx}$ ) were measured continuously before, during, and after CSF removal. CSF was removed by the neonatologist either by lumbar puncture or by accessing a ventricular reservoir. Once CSF was obtained in the needle hub, the infants were placed in a relaxed, extended position to obtain an accurate opening pressure. The time of the start and end of CSF removal was marked in the NIRO500 tracing with an event marker.

Data analysis. For each CSF removal procedure, the changes in the concentration of each chromophore were calculated as follows. We calculated the means of 10 sequential data points obtained just before and after CSF removal to provide the pre- and post-CSF removal measurements for $\mathrm{HbO}$ and $\mathrm{Hb}$. A mean of 10 points was calculated because there was some minimal variability in the NIRS signals, even in the absence of any physiologic or pathologic changes in the infant's clinical condition. In this study, the variability in the NIRS signals in the absence of any apparent real or artifactual perturbations was $0.1-0.3 \mu \mathrm{M}$ for the $\mathrm{Hb}$ signals and $0.05-$ $0.15 \mu \mathrm{M}$ for the CytOx signal (the magnitude of the CytOx signal is much smaller than that of the $\mathrm{HbO}$ and $\mathrm{Hb}$ signals). The means for $\mathrm{HbT}$ and $\mathrm{HbD}$ were derived from the sum and difference, respectively, of the $\mathrm{HbO}$ and $\mathrm{Hb}$ signals. Thus the total change in any particular NIRS signal caused by CSF removal was calculated as the difference between these preand post-CSF removal mean values.

Data were excluded from analysis if there was excessive movement artifact in the recording, because movement of the subject produces artifactual changes in NIRS signals. At the time of study, any movements of the baby were recorded in the NIRS data with an event marker. If there were large $(>2 \mu \mathrm{M})$, abrupt $(<2 \mathrm{~s}$ duration) changes in NIRS signals that coincided with the baby's movements, the data were excluded as these changes do not reflect true changes in cerebral hemodynamics related to CSF removal. Also, data were excluded from the analysis if there was an oxygen desaturation to less than $87 \%$ during the study, as such a desaturation also produces a significant change in NIRS signals that cannot be attributed only to CSF removal.

Finally, a linear regression was performed to determine whether there was any correlation between the change in each of these NIRS signals (e.g. $\mathrm{HbO}, \mathrm{Hb}, \mathrm{HbD}, \mathrm{HbT}$ ) resulting from CSF removal and either the volume of CSF removed or intracranial pressure. The change in the infant's CBV was determined by the change in $\mathrm{HbT}$, according to the relationship $\Delta \mathrm{CBV}=\Delta \mathrm{HbT} \times 0.89 /[\mathrm{Hgb}]$, where $[\mathrm{Hgb}]$ is the infant's serum $\mathrm{Hb}$ concentration, and 0.89 is a constant that reflects the large vessel to cerebral hematocrit ratio, the brain density in grams per milliliter, and the molecular weight of hemoglobin (22).

Statistical analysis. To determine whether there was a statistically significant change in a particular NIRS signal with CSF removal, a paired $t$ test was used to compare the mean value before and after CSF removal for each NIRS signal. Analysis of the correlation between NIRS signal changes and 
opening pressure or CSF volume removed was performed using linear regression analysis. All data were analyzed using Sigmastat software (SPSS Inc., Chicago, IL, U.S.A.).

\section{RESULTS}

A total of 32 studies of NIRS changes during CSF removal were made on 16 infants with $\mathrm{PHH}$. Of these, nine studies on six infants were excluded because of movement artifact, leaving 23 studies on 12 infants that were analyzed. The clinical characteristics of the infants are shown in Table 1. Multiple studies on the same infant were performed at least $24 \mathrm{~h}$ apart. The gestational age at birth of the infants was $29 \pm 3.9 \mathrm{wk}$ (mean $\pm \mathrm{SD}$; range, 25-38 wk), and the gestational age at the time of NIRS studies was $33 \pm 3 \mathrm{wk}$ (range, 27-40 wk). The volume of CSF removed was $8.6 \pm 3.4 \mathrm{~mL} / \mathrm{kg}$ body weight (range, $2.9-15 \mathrm{~mL} / \mathrm{kg}$ ), and the opening pressure measured at the time of CSF removal was $144 \pm 42 \mathrm{~mm} \mathrm{H}_{2} \mathrm{O}$ (range, 65-220 $\mathrm{mm} \mathrm{H}_{2} \mathrm{O}$ ), measured by manometry.

NIRS changes during CSF removal. The change in NIRS signals with CSF removal was analyzed by comparing baseline NIRS signal values with values at the end of CSF removal. There was a statistically significant increase in $\mathrm{HbO}, \mathrm{HbT}$, and $\mathrm{HbD}$ with $\mathrm{CSF}$ removal but no significant change in $\mathrm{Hb}$ (Fig. $1)$. The mean change in $\mathrm{HbO}$ was $4.21 \pm 0.82 \mu \mathrm{M}$ (mean \pm $\mathrm{SEM}, p<0.001)$, the change in $\mathrm{HbT}$ was $4.48 \pm 1.2 \mu \mathrm{M}(p$ $=0.001)$, and the change in $\mathrm{HbD}$ was $3.94 \pm 1.30 \mu \mathrm{M}(p=$ 0.006). The change in $\mathrm{HbT}$ corresponded to an increase in CBV of $0.44 \pm 0.10 \mathrm{~mL} / 100 \mathrm{~g}$ accompanying CSF removal ( $\Delta \mathrm{CBV}$ calculated from $\Delta \mathrm{HbT}$ as outlined in "Methods"). There was no significant change in CytOx with CSF removal (Fig. 1).

Relationship between change in NIRS signals and opening pressure or CSF volume removed. Next, we examined the relationship between the changes in NIRS signals (specifically, $\mathrm{HbD}$ and $\mathrm{HbT}$ ) and the volume of CSF removed and the opening CSF pressure. There was no statistically significant correlation between $\Delta \mathrm{HbD}$ or $\Delta \mathrm{HbT}$ and either the volume of CSF removed (in milliliters per kilogram of body weight) or the opening pressure measured at time of CSF removal (data not shown). There was an insufficient number of measurements of the closing pressure to compare the change in NIRS signals with the change in intracranial pressure.

\section{DISCUSSION}

The principal finding of this study is the significant increase in $\mathrm{HbO}, \mathrm{HbD}$, and $\mathrm{HbT}$ (and thus $\mathrm{CBV}$ ) that resulted from CSF removal in infants with $\mathrm{PHH}$. This finding demonstrates that CSF removal in infants with $\mathrm{PHH}$ has an important and potentially beneficial effect on cerebral hemodynamics. The increase of $0.44 \pm 0.10 \mathrm{~mL} / 100 \mathrm{~g}$ in the CBV reflects a $20 \%$ increase in $\mathrm{CBV}$, as $\mathrm{CBV}$ has been measured previously to be 2.2-2.5 $\mathrm{mL} / 100 \mathrm{~g}$ in the newborn infant (determined by NIRS or positron emission tomography) $(23,24)$. Moreover, this increase in $\mathrm{CBV}$ is likely to be due to an increase in $\mathrm{CBF}$, because the change in the sum of $\mathrm{HbO}$ and $\mathrm{Hb}$ is caused by the increase in $\mathrm{HbO}$, the principal chromophore of the arterial compartment. Notably, changes in HbD were shown to reflect changes in CBF (measured simultaneously by radioactive microspheres) in a piglet model of hydrocephalus (17). Thus the finding of an increase in $\mathrm{HbD}$ accompanying CSF removal in human infants further supports the conclusion that this increase reflects an increase in CBF. This apparent increase in CBF with CSF removal is consistent with animal studies showing 1) that treatment of induced hydrocephalus (by early shunt placement) results in reversal of the decrease in CBF and capillary density caused by the hydrocephalic process $(10,11)$, and 2) that induced acute hydrocephalus results in the opposite finding, namely reductions in $\mathrm{HbO}$ and $\mathrm{HbD}$ and in $\mathrm{CBF}(17,25)$.

We also addressed whether there was an increase in the oxidized form of CytOx accompanying CSF removal, suggesting an improvement in intracellular oxidative metabolism (and not only an increase in intravascular oxygenation). In fact, we found no significant change in CytOx accompanying CSF removal. The only previous published study using NIRS in infants with $\mathrm{PHH}$ showed that three of six infants studied had an increase in oxidized CytOx accompanying CSF removal (changes in $\mathrm{HbO}, \mathrm{Hb}$, and so forth were not reported) (26). One explanation for this discrepancy may be a difference in the severity or duration of progressive ventriculomegaly in the two studies. Animal studies of hydrocephalus have shown that a

Table 1. Table of clinical characteristics of 12 patients with reported NIRS measurements

\begin{tabular}{|c|c|c|c|c|c|c|c|}
\hline $\begin{array}{c}\text { Subject } \\
\text { no. }\end{array}$ & $\begin{array}{c}\text { Birth GA } \\
\text { (wk) }\end{array}$ & $\begin{array}{c}\text { Birth weight } \\
\text { (g) }\end{array}$ & $\begin{array}{l}\text { IVH grade } \\
\text { (L-R)* }\end{array}$ & $\begin{array}{c}\text { Age at } \\
\text { onset VD } \\
\text { (d) }\end{array}$ & $\begin{array}{l}\text { Age at } 1 \text { st } \\
\text { NIRS study } \\
\text { (d) }\end{array}$ & $\begin{array}{l}\text { Age at serial taps } \\
\text { (1st-last) } \\
\text { (d) }\end{array}$ & $\begin{array}{c}\text { Total no. } \\
\text { of taps }\end{array}$ \\
\hline 1 & 26 & 810 & 3 & 3 & 17 & $17-33$ & 4 \\
\hline 3 & 24 & 690 & 2 & 5 & 34 & $31-36$ & 4 \\
\hline 4 & 30 & 1600 & $2-3$ & 10 & 22 & $18-64$ & 32 \\
\hline 5 & 26 & 900 & $2-3$ & 5 & 16 & $16-76$ & 8 \\
\hline 8 & 27 & 1205 & 3 & 11 & 25 & $14-49$ & 13 \\
\hline 9 & 29 & 1530 & 3 & 7 & 12 & $12-25$ & 3 \\
\hline 10 & 38 & 3300 & 3 & 5 & 18 & $14-29$ & 3 \\
\hline 11 & 26 & 715 & 2 & 69 & 70 & $70-81$ & 4 \\
\hline 12 & 28 & 1030 & 3 & 3 & 23 & $23-54$ & 4 \\
\hline
\end{tabular}

* Two grades of IVH were recorded when the grades differed on left (L) and right (R) sides [IVH grading scheme per Volpe (1)].

Abbreviations used: Age at onset VD, age in days when progressive ventricular dilation was first noted by cranial ultrasound; GA, gestational age. 


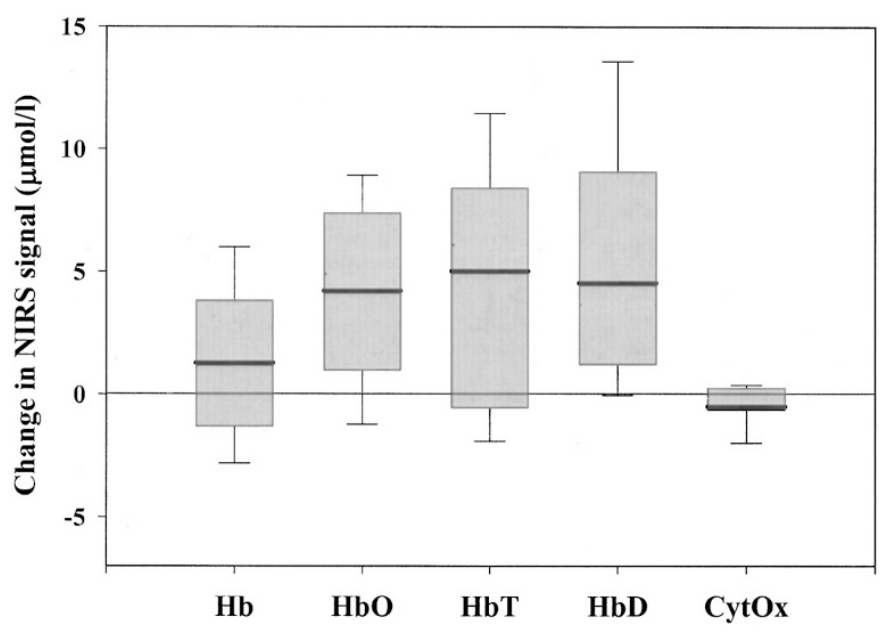

Figure 1. Box plot showing the change in NIRS signals (in micromoles per liter) accompanying CSF removal. The box edges represent the 25th and 75th percentiles, the thick line within the box is the mean, and the error bars represent the fifth and 95th percentiles.

reduction in $\mathrm{CBF}$ occurs before a reduction in oxidative metabolism and irreversible injury (27). Thus detection of impaired cerebral hemodynamics may be critical for identifying infants at imminent risk for failure of oxidative metabolism and the subsequent development of irreversible parenchymal injury.

One possible explanation for the data obtained is that the changes in NIRS signals measured were the result of normal variation. We did not have a separate control group for comparison in this study; however, we used NIRS measurements made before and after the CSF removal procedure as control NIRS data for each infant. All infants in this study underwent at least $10 \mathrm{~min}$ (often $20-30 \mathrm{~min}$ ) of stable NIRS recording before or after CSF removal, during which time there was minimal variability in the signals measured. In this way, each subject served as his or her own control for the study. We found that the typical normal variability was on the order of 2 to $8 \%$ of the magnitude of changes in NIRS signals accompanying CSF removal. In this and other NIRS studies we have conducted, we have found that large changes in NIRS signals are typically related to significant physiologic or pathologic perturbations. These perturbations would include events such as apnea or bradycardia with significant oxygen desaturation, large changes in blood pressure, or manipulations of ventilator settings resulting in significant changes in $\mathrm{PCO}_{2}$. Similarly, large artifactual changes in NIRS signals may result from an infant's movements (a well-known limitation of the NIRS technique). It is unknown whether the NIRS signals return to the pre-event values after a perturbation such as an apnea with desaturation or movement artifact, hence the decision to exclude data containing such events. We do not think that exclusion of such data was a source of significant bias in this study, as such events did not appear to correlate with the severity of PHH or other clinical features in this small group of infants. Thus we are confident that the changes in NIRS signals reported in this study reflect changes in cerebral hemodynamics resulting from CSF removal in infants with $\mathrm{PHH}$.
The specific features of PHH responsible for the effects on cerebral hemodynamics are not clear. The major possibilities include the degree of ventriculomegaly, the degree of intracranial pressure elevation, or both. Our study showed no statistically significant relationship between the changes in NIRS signals $(\mathrm{HbD}$ and $\mathrm{HbT})$ and the opening pressure measured or the volume of CSF removed; this lack of correlation may be related to our relatively small sample size. Because our study design did not include quantitation of ventriculomegaly or closing intracranial pressure, we could not accurately correlate the increase in $\mathrm{HbO}, \mathrm{HbT}$, and $\mathrm{HbD}$ with the degree of changes in ventricular size or intracranial pressure.

\section{CONCLUSIONS}

The results obtained in this study show a pronounced effect of CSF removal on cerebral hemodynamics in infants with PHH. These data support the value of CSF removal (even when intermittent) in the treatment of $\mathrm{PHH}$ in improving cerebral perfusion. The findings further underscore the difficulty of using opening pressure or volume of CSF removed to guide therapy, as the effect of CSF removal on cerebral perfusion could not be predicted by these clinical measures. The difficulty of obtaining NIRS data during CSF removal that is free of movement artifact and other perturbations (e.g. changes with apnea) may limit the ability of this particular application of NIRS to detect clinically important changes in cerebral perfusion with CSF removal in PHH. However, NIRS is uniquely suited to repeated, noninvasive bedside measurements of cerebral hemodynamics in infants with conditions such as PHH that may disturb cerebral perfusion. NIRS may prove to be valuable in detecting impending cerebral ischemia and thereby guiding appropriate therapy of infants with $\mathrm{PHH}$.

Acknowledgments. The authors thank Shaye Moore for assistance in manuscript preparation.

\section{REFERENCES}

1. Volpe JJ 2001 Neurology of the Newborn. WB Saunders Co., Philadelphia, pp 428-496

2. Dykes FD, Dunbar B, Lazarra A, Ahmann PA 1989 Posthemorrhagic hydrocephalus in high-risk preterm infants: natural history, management, and long-term outcome. J Pediatr 114:611-618

3. Msall ME, Buck GM, Rogers BT, Merke D, Catanzaro NL, Zorn WA 1991 Risk factors for major neurodevelopmental impairments and need for special education resources in extremely premature infants. J Pediatr 119:606-614

4. Resch B, Gedermann A, Maurer U, Ritschl E, Muller W 1996 Neurodevelopmental outcome of hydrocephalus following intra-/periventricular hemorrhage in preterm infants: short- and long-term results. Childs Nerv Syst 12:27-33

5. International PHVD Drug Trial Group 1998 International randomised controlled trial of acetazolamide and furosemide in posthaemorrhagic ventricular dilatation in infancy. Lancet 352:433-440

6. Ventriculomegaly Trial Group 1990 Randomized trial of early tapping in neonatal posthemorrhagic ventricular dilation. Arch Dis Child 65:3-10

7. du Plessis AJ 1998 Posthemorrhagic hydrocephalus and brain injury in the preterm infant: dilemmas in diagnosis and management. Semin Pediatr Neurol 5:161-179

8. de Vries LS, Liem KD, van Dijk K, Smit BJ, Sie L, Rademaker KJ, Gavilanes AW 2002 Early versus late treatment of posthaemorrhagic ventricular dilatation: results of a retrospective study from five neonatal intensive care units in The Netherlands. Acta Paediatr 91:212-217

9. da Silva M, Drake J, Lemaire C, Cross A, Tuor U 1994 High-energy phosphate metabolism in a neonatal model of hydrocephalus before and after shunting. J Neurosurg 81:544-553

10. da Silva MC, Michowicz S, Drake JM, Chumas PD, Tuor UI 1995 Reduced local cerebral blood flow in periventricular white matter in experimental neonatal hydrocephalus-restoration with CSF shunting. J Cereb Blood Flow Metab 15:1057-1065 
11. Del Bigio M, Bruni J 1988 Changes in periventricular vasculature of rabbit brain following induction of hydrocephalus and after shunting [published erratum appears in J Neurosurg 1988;69:963]. J Neurosurg 69:115-120

12. Del Bigio MR, Kanfer JN, Zhang YW 1997 Myelination delay in the cerebral white matter of immature rats with kaolin-induced hydrocephalus is reversible. J Neuropathol Exp Neurol 56:1053-1066

13. del Bigio MR, Crook CR, Buist R 1997 Magnetic resonance imaging and behavioral analysis of immature rats with kaolin-induced hydrocephalus: pre- and postshunting observations. Exp Neurol 148:256-264

14. Hill A, Volpe JJ 1982 Decrease in pulsatile flow in the anterior cerebral artery in infantile hydrocephalus. Pediatrics 69:4-7

15. Taylor GA, Phillips MD, Ichord RN, Carson BS, Gates JA, James CS 1994 Intracranial compliance in infants: evaluation with Doppler US. Radiology 191:787-791

16. Tsuji M, du Plessis A, Taylor G, Crocker R, Volpe JJ 1998 Near infrared spectroscopy detects cerebral ischemia during hypotension in piglets. Pediatr Res 44:591-595

17. Soul JS, Taylor GA, Wypij D, du Plessis AJ, Volpe JJ 2000 Noninvasive detection of changes in cerebral blood flow by near-infrared spectroscopy in a piglet model of hydrocephalus. Pediatr Res 48:445-449

18. Soul JS, du Plessis AJ 1999 New technologies in pediatric neurology. Near-infrared spectroscopy. Semin Pediatr Neurol 6:101-110

19. Wray S, Cope M, Delpy D, Wyatt J, Reynolds E 1988 Characterization of the nea infrared absorption spectra of cytochrome aa 3 and haemoglobin for the non-invasive monitoring of cerebral oxygenation. Biochim Biophys Acta 933:184-192
20. Wyatt J, Delpy DT, Cope M, Wray S, Reynolds E 1986 Quantification of cerebral oxygenation and hemodynamics in sick newborn infants by near infrared spectroscopy. Lancet 2:1063-1066

21. Wyatt J, Cope M, Delpy D, van der Zee P, Arridge S, Edwards A, Reynolds E 1990 Measurement of optical path length for cerebral near-infrared spectroscopy in newborn infants. Dev Neurosci 12:140-144

22. Wyatt JS, Edwards AD, Cope M, Delpy DT, McCormick DC, Potter A, Reynolds EO 1991 Response of cerebral blood volume to changes in arterial carbon dioxide tension in preterm and term infants. Pediatr Res 29:553-557

23. Wyatt JS, Cope M, Delpy DT, Richardson CE, Edwards AD, Wray S, Reynolds EO 1990 Quantitation of cerebral blood volume in human infants by near-infrared spectroscopy. J Appl Physiol 68:1086-1091

24. Altman DI, Perlman JM, Volpe JJ, Powers WJ 1993 Cerebral oxygen metabolism in newborns. Pediatrics 92:99-104

25. Proctor HJ, Cairns C, Fillipo D, Palladino GW, Rosner MJ 1984 Brain metabolism during increased intracranial pressure as assessed by niroscopy. Surgery 96:273-279

26. Casaer P, von Siebenthal K, van der Vlugt A, Lagae L, Devlieger H 1992 Cytochrome $a a_{3}$ and intracranial pressure in newborn infants; a near infrared spectroscopy study. [letter] Neuropediatrics 23:111

27. Richards HK, Bucknall RM, Jones HC, Pickard JD 1995 Uncoupling of 1CBF and 1CGU in two different models of hydrocephalus: a review. Childs Nerv Syst 11:288292 